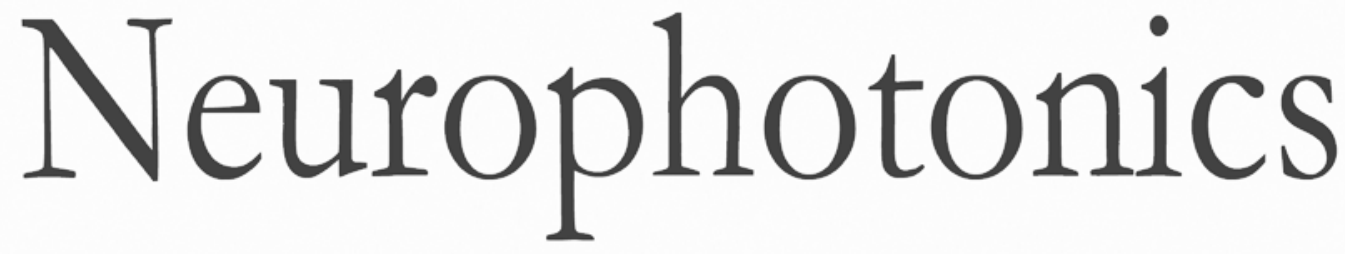

\title{
'Blue' voltage-sensitive dyes for studying spatiotemporal dynamics in the brain: visualizing cortical waves
}

\author{
Xinling Geng \\ Jian-Young $\mathrm{Wu}$
}




\title{
'Blue' voltage-sensitive dyes for studying spatiotemporal dynamics in the brain: visualizing cortical waves
}

\author{
Xinling Geng ${ }^{\mathrm{a}, \mathrm{b}}$ and Jian-Young $\mathrm{Wu}^{\mathrm{b}, *}$ \\ ${ }^{a}$ Capital Medical University, School of Biomedical Engineering, Department of Biomedical Instrumentation, Beijing, China \\ ${ }^{\mathrm{b}}$ Georgetown University, Department of Neuroscience, Washington, DC, United States
}

\begin{abstract}
Among many distinct contributions made by Amiram Grinvald's group, the "Blue dyes" is a special gift for visualizing cortical population neuronal activity. The excitation wavelength of blue dyes has minimal overlap with the absorption of hemoglobin, and hence has minimal pulsation artifacts. This advantage leads to high signal-to-noise ratio optical recordings of cortical activity, with sensitivity as good as that of local field potential recordings. High sensitivity imaging allows for recording of spontaneous and evoked activity in single trials without spatial or temporal averaging, and has benefitted many scientists in their research projects. Single trial recording is particularly important for studying the cortex, because spontaneous and ongoing activities interact with sensory evoked events, creating rich dynamics in the wave patterns. Signal averaging in space and time would diminish the dynamic components in the patterns. Here, we discuss how the blue dyes help to achieve high-sensitivity voltage-sensitive dye imaging of spontaneous and evoked cortical activities. Spontaneous cortical activity has a constantly changing spatial pattern and temporal frequency, making it impossible to average in space and time. Amiran Grinvald's invention of blue dyes made it possible to examine the spatiotemporal patterns of cortical dynamics, about 15 years before the first useful genetically coded voltage proteins became available. $\odot$ The Authors. Published by SPIE under a Creative Commons Attribution 3.0 Unported License. Distribution or reproduction of this work in whole or in part requires full attribution of the original publication, including its DOI. [DOI: 10.1117/1.NPh.4.3.031207]
\end{abstract}

Keywords: blue voltage-sensitive dyes; spatiotemporal dynamics; cortical waves.

Paper 16071SSR received Dec. 5, 2016; accepted for publication Feb. 13, 2017; published online Mar. 9, 2017.

\section{Introduction}

The "blue" voltage-sensitive dyes (VSD) referred to a special kind of dyes, namely RH1691, RH1692, and RH1838, developed by Amiram Grinvald's group. ${ }^{1}$ Among many contributions to neuroscience achieved by the Grinvald group, the blue dyes have advanced the imaging of the mammalian cortex. ${ }^{2-10}$ The excitation wavelength of blue dyes has minimal overlap with the absorption of hemoglobin, and hence has minimal pulsation artifacts. ${ }^{1}$ This advantage leads to high signal-to-noise ratio optical recordings of cortical activity, with sensitivity as good as that of local field potential (LFP) recordings. ${ }^{2}$ This sensitivity allows for recording of spontaneous and evoked activity in single trials without spatial or temporal averaging.

Here, we discuss mainly our own practice $e^{2,7-10}$ on imaging spontaneous and evoked cortical activities to elaborate the importance of using blue dyes for high signal-to-noise recordings in the mammalian cortex.

\section{Staining the Cortex with the Blue Dyes}

Labeling cortical neurons is important for good VSD signals. Methods were discussed in detail in our previous publication. ${ }^{2}$ In brief, dye solution needs to be gently perfused on the surface of the cortex for about $2 \mathrm{~h}$. Long staining time is needed for a well-distributed labeling down into layers II-III [Figs. 1(a) and 1(b)]. It is also essential not to irritate the cortex during dissection and subsequent staining procedures. We found that even

*Address all correspondence to: Jian-Young Wu, E-mail: wuj@ georgetown.edu minor irritation, seen as slight redness of the pia capillaries, may significantly block the staining of the cortex. In order to protect the cortex from irritation, we did not remove the dura for staining and imaging. Keeping the dura intact also greatly reduces the movement of the cortex caused by pulsation and respiration. From the staining profile along the depth of the cortex [Figs. 1(a) and 1(b)], we estimated that most of the VSD signals are from upper layer II-III, up to $\sim 400-\mu$ m deep from the pia surface. VSD signals decline with recording time, probably due to bleaching of the dye. The useful imaging time is about $1000 \mathrm{~s}$ under high intensity light exposure [Fig. 1(a)]. Here, "high intensity" refers to an illumination level under which most of the peaks in the LFP show a corresponding event in the VSD trace [Fig. 2(d)]. On our recording apparatus, this "high" illumination intensity is generated by an $\sim 80$-W halogen lamp, filtered by a $630 / 30-\mathrm{nm}$ filter, and projected to the cortex via a $5 \times(0.4 \mathrm{NA})$ macroscope. Reduction of the illuminating intensity would allow longer recording time ( 1 to $2 \mathrm{~h}$ ). After long light exposure, while the VSD signal is largely bleached, a shielded area next to the exposed area immediately shows near normal VSD amplitude [Fig. 1(d)], suggesting that the blue dyes have ignorable phototoxicity.

\section{High Sensitivity Optical Recordings}

Spontaneous cortical activity has a constantly changing spatial pattern and temporal frequency, making it impossible to average in space and multiple trials. ${ }^{2,7-11}$ The VSD signals of spontaneous cortical activity are about $0.1 \%$ of the resting light intensity [Fig. 2(b)], much smaller than the $\sim 0.5 \%$ of pulsation artifacts 
(a)

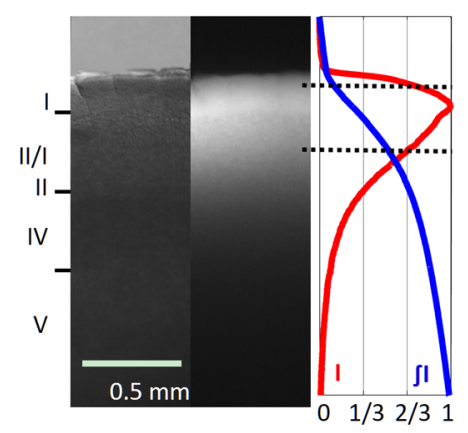

(b)

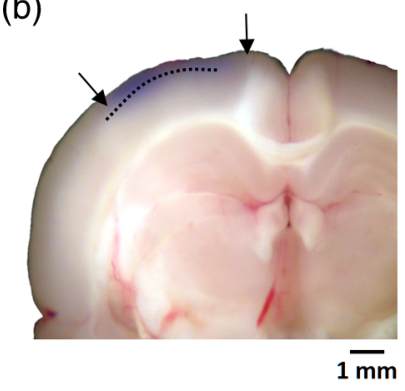

(c)

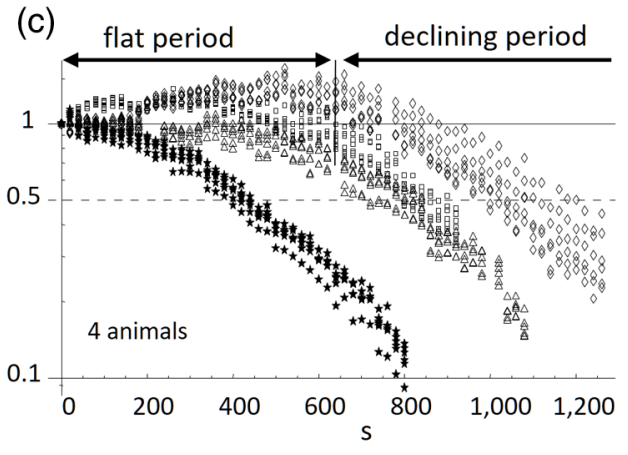

(d)

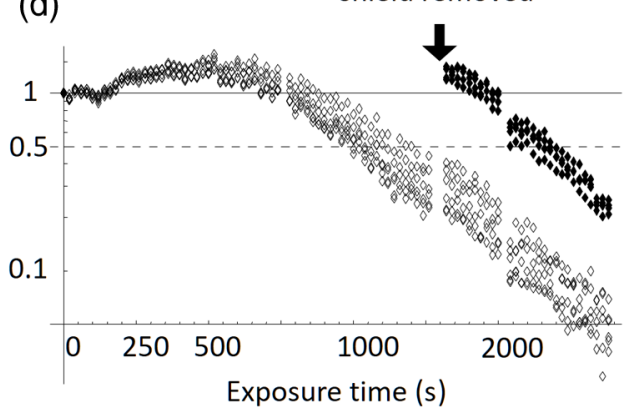

Fig. 1 (a) Left, visible light image of the stained cortex. Middle, fluorescence image from the same cortical section showing distribution of RH1691 staining. Right, fluorescence intensity (red) and integrated fluorescence intensity (blue). Broken lines mark the tissue depth with fluorescent intensity higher than two-thirds of the maximum intensity. (b) Photograph of a stained cortical section. Arrows indicate the boundary of the cranial window. The broken line indicates the border between gray and white matter. Staining of RH1691 can be seen by eye as a light blue hue. (c) Normalized VSD signal (bicuculline induced spikes) plotted against light exposure time. Data from three animals with good staining (diamonds, triangles, squares) and one with poor staining (stars). Note that the signal amplitude remained stable (or slightly increased) during the flat period. After the flat period, the signal had a higher rate of decline with light exposure (declining period). (d) Data from another animal, in which part of the cortex was shielded from light exposure. In the shielded area, the signal amplitude remained unchanged after long light exposure (solid diamonds), while signal in unshielded area declined (empty diamonds), suggesting that the signal decline was related to light exposure.

of a nonblue dye RH795 [Fig. 2(a)]. The reduction of pulsation artifacts is thus essential for high-sensitivity single trial recordings. Pulsation artifacts bring two interferences to VSD imaging. One is the movement of the tissue with the pulse, which can be greatly reduced by sealing the recording chamber and movement subtraction algorithms (described in detail in Fig. 5 of Lippert et al. ${ }^{2}$ ). The other is the change in hemoglobin absorption with the pulse, which can be removed only by application of the RH1691 or RH1838 [Figs. 2(a) and 2(b)]. Removing pulsation artifacts greatly enhances the signal-to-noise ratio of VSD recordings, and the sensitivity of VSD recordings can be as good as that of LFP recordings [Figs. 2(c) and 2(d)]. We used a photodiode array for all our measurements described in this article. The diode array has an effective dynamic range of 19 to 21 bit, allowing measurement of small signals between $0.01 \%$ to $0.1 \%$ change in the baseline fluorescent light (recently reviewed by Liang et al. ${ }^{11}$ ). When cortical activity by VSD imaging and LFP was simultaneously recorded from the same tissue, $>60 \%$ of the peaks in the LFP showed a corresponding event in the VSD trace [Figs. 2(c) and 2(d)]. Under 1.5\% isoflurane anesthesia, infrequent bursts of spontaneous activity occurred on both electrical and optical recordings [Fig. 2(c)]. However, LFP and VSD signals were frequently disproportionate in amplitude. In Fig. 2(c), events labeled with dots had higher amplitude in VSD trace compared to those in the LFP trace, while events labeled with triangles had larger amplitude in the LFP trace compared to those in the VSD trace. Events labeled with diamonds were seen in the LFP but not in the VSD signals. These may be caused by the different origins of the LFP and VSD signals, e.g., LFP signals may be generated in the deep cortical layers, not seen by VSD imaging. When the level of anesthesia was lowered to $\sim 1.1 \%$, both VSD and LFP signals showed continuous spontaneous fluctuations [Fig. 2(d)]. The fluctuations in the VSD signals are likely to be biological signals because the baseline noise is much smaller in the quiescent segments of Fig. 2(c). Since almost every event in the LFP can be seen in the VSD recordings, the sensitivity of the VSD measurement is comparable to that of the LFP recordings. The correlation between optical traces is high and each peak in the signal appears at many locations [Figs. 2(c) and 2(d), bottom traces]. High correlation between optical traces is not from light scattering or optical blurring between channels, because there is a small timing difference between locations, resulting in a globally propagating wave.

\section{Trial-to-Trial Variations}

Blue dyes allow for obtaining high signal-to-noise ratio data from hundreds of trials from each animal. These kinds of 
(a) Conventional dye (RH795)

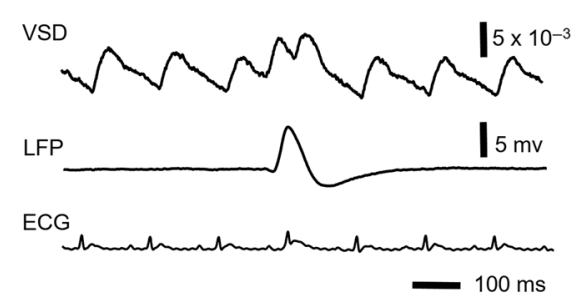

(b) "Blue" dye (RH1691)

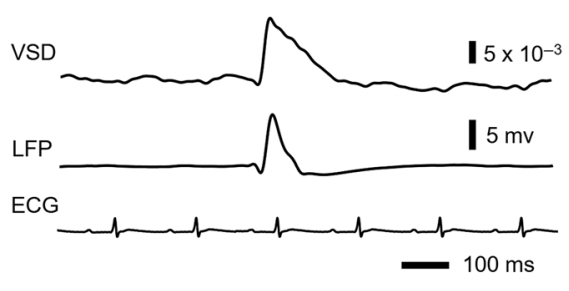

(c) Spontaneous burst (1.5\% isoflurane)

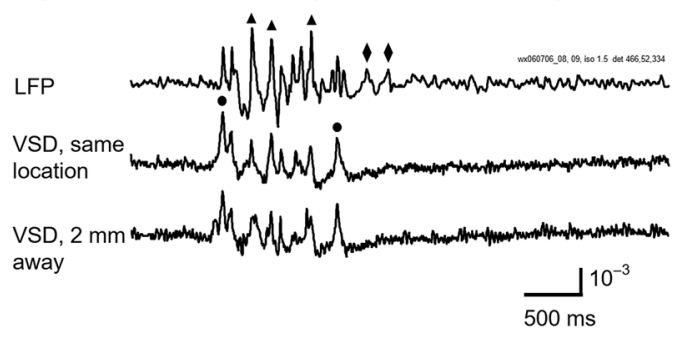

(d) Spontaneous oscillations (1.1\% isoflurane)

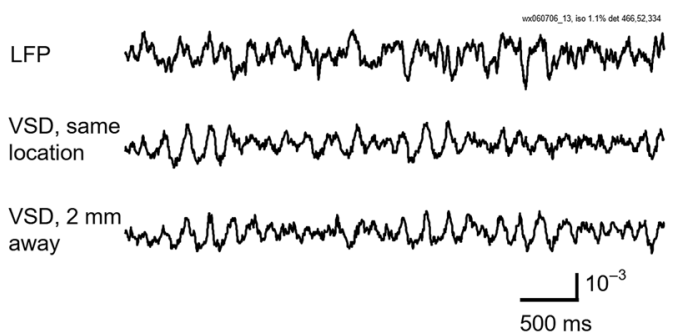

Fig. 2 Pulsation artifact and the sensitivity of blue dyes. (a) and (b) Optical recordings (VSD) from cortex stained with conventional dye RH795 (a) and the blue dye RH1691 (b). LFP in cortex and electrocardiogram (ECG) are simultaneously recorded. Epileptiform spikes induced by bicuculline were used as the signal source of neuronal activity. The pulsation artifact is much smaller in blue dye signals compared to conventional VSD. (c) and (d) Spontaneous cortical activities under different levels of anesthesia, demonstrating the sensitivity of blue dye recordings. Note that the scale is one-fifth of that in (a) and (b), demonstrating that the pulsation artifact of the conventional VSD is approximately five times of that of spontaneous neuronal activity. Modified from Ref. 2.

(a) Barrel cortex

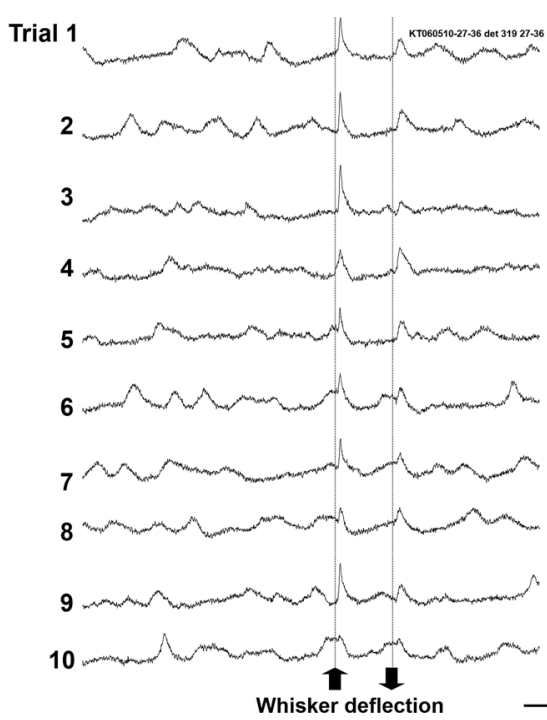

(b) Visual cortex

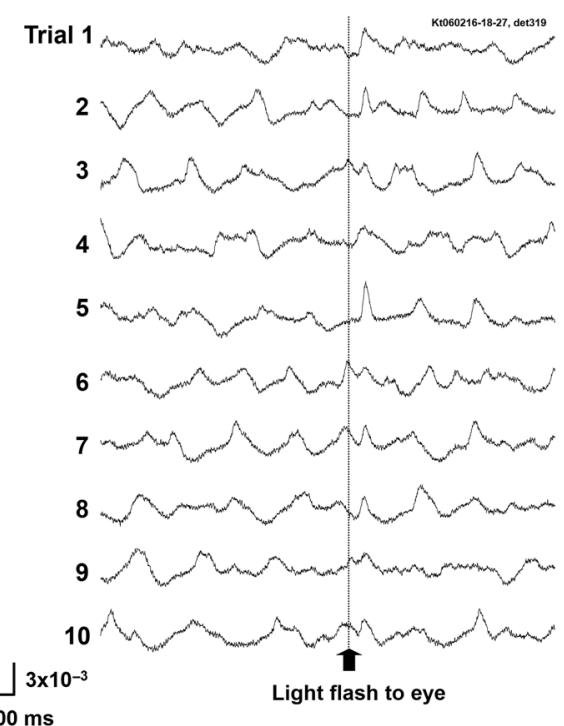

Fig. 3 Evoked cortical activity from (a) rat barrel and (b) visual cortex. Ten recording trials with identical stimulus intensity demonstrate large trial-to-trial variations on the background of ongoing cortical activity. Modified from Ref. 2.

data allow for examining trial-to-trial variations of cortical activity evoked by identical sensory stimuli (Figs. 3 and 4). The amplitude of the evoked response fluctuates with the spontaneous activity [Fig. 3(a), traces 9, 10; Fig. 3(b), traces 5, 6], suggesting the trial-to-trial variations of the evoked activity may be caused by interactions with the spontaneous, ongoing activity.

Variations in evoked cortical activity also occurred in spatial pattern. Both spontaneous and evoked cortical activity manifested as propagating waves (Fig. 4). Sensory evoked waves 


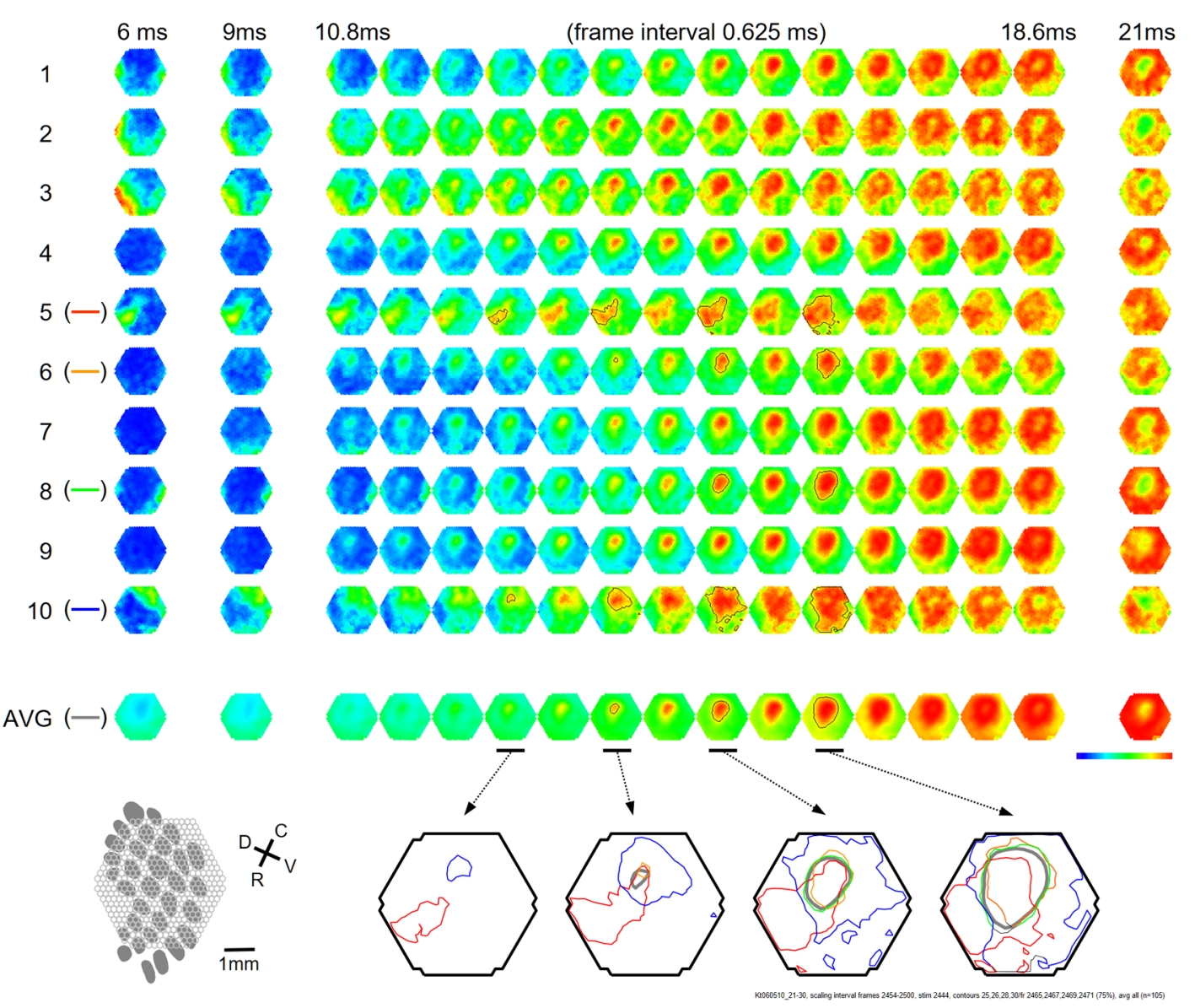

Fig. 4 Trial-to-trial variations in barrel cortex. Image rows 1 to 10: Ten consecutive trials with identical whisker deflection. The bottom images (AVG) were averaged from 105 trials from the same animal. Bottom row left: schematic diagram of the barrel pattern and the imaging field. Bottom row right: isochromatic contour lines were superimposed from trials 5 (red), 6 (orange), 8 (green), and 10 (blue), along with contour lines from the averaged data (gray). Modified from Ref. 2.

followed a consistent propagation pattern, initiating from the location of the thalamic afferents and spreading to large areas of the cortex. In barrel cortex, whisker evoked waves initiated from within the corresponding barrel and propagated to the entire barrel cortex. In images obtained from multiple trial averaging, this general propagating pattern can be nicely seen (Fig. 4, image row AVG). An overall propagating velocity of $0.2 \pm 0.1 \mathrm{~m} / \mathrm{s}, \quad(n=6$ animals $)$ was obtained. However, when single trial data were examined, the propagating velocity varied largely, ranging from 0 (no propagation) to about $200 \mathrm{~mm} / \mathrm{s}$. As shown in Fig. 4, large trial-to-trial variations were observed in the propagating patterns. The propagation pattern of individual trials was compared with that of the multiple trial averages. While all trials showed a similar overall spatiotemporal pattern, significant trial-to-trial variations can be seen in individual trials. In the bottom row of Fig. 4 , the contour lines of the propagation patterns for trials $5,6,8$, and 10 were superimposed, in order to compare them with the contours of the average pattern. The contour lines were highly variable, suggesting that the propagation of an evoked wave was highly dynamic. Since spontaneous waves occurred frequently, they may interact with the evoked response and contribute to anisotropic propagation patterns. In trial 5, the evoked activity appeared to merge with a spontaneous event and propagated faster in the direction toward the spontaneous event.

\section{Compression and Reflection of Visually Evoked Waves}

Using blue dyes, sensory evoked cortical waves can be examined in a greater detail from the mammalian cortex. A lower pulsation artifact allows a dynamic range of 17 to 19 bit.

Propagating waves (a.k.a. traveling waves) of neuronal activity are extensively seen in the cortex..$^{6,7,12-26}$ A sensory-evoked propagating wave starts at the cortical sensory representation and propagates into a larger area including the whole primary and secondary cortical areas. A number of mechanisms have been proposed for cortical waves. ${ }^{27,28}$

A drifting grating $(0.05$ cycle $/ \mathrm{deg}, 50 \mathrm{w} \times 38 \mathrm{~h}$ deg of viewing angle) presented to the contralateral eye can reliably evoke a propagating wave in the visual cortex (Fig. 5). The activity was seen in all optical detectors with a small time difference between each detector [Fig. 5(a), traces 1 to 4]. The signal on each individual detector was converted to pseudocolor according to a linear color scale. The pseudocolor images showed that the evoked wave initiated in the monocular area of V1 (V1M) and propagated in both directions to the V1 binocular area (V1B) and to V2. The wave was "compressed" in its spatial dimension into a thin band in the middle of the propagating path [Figs. 5(a) and 5(b) images]. A reflected wave was initiated after compression and propagated backward to V1. 

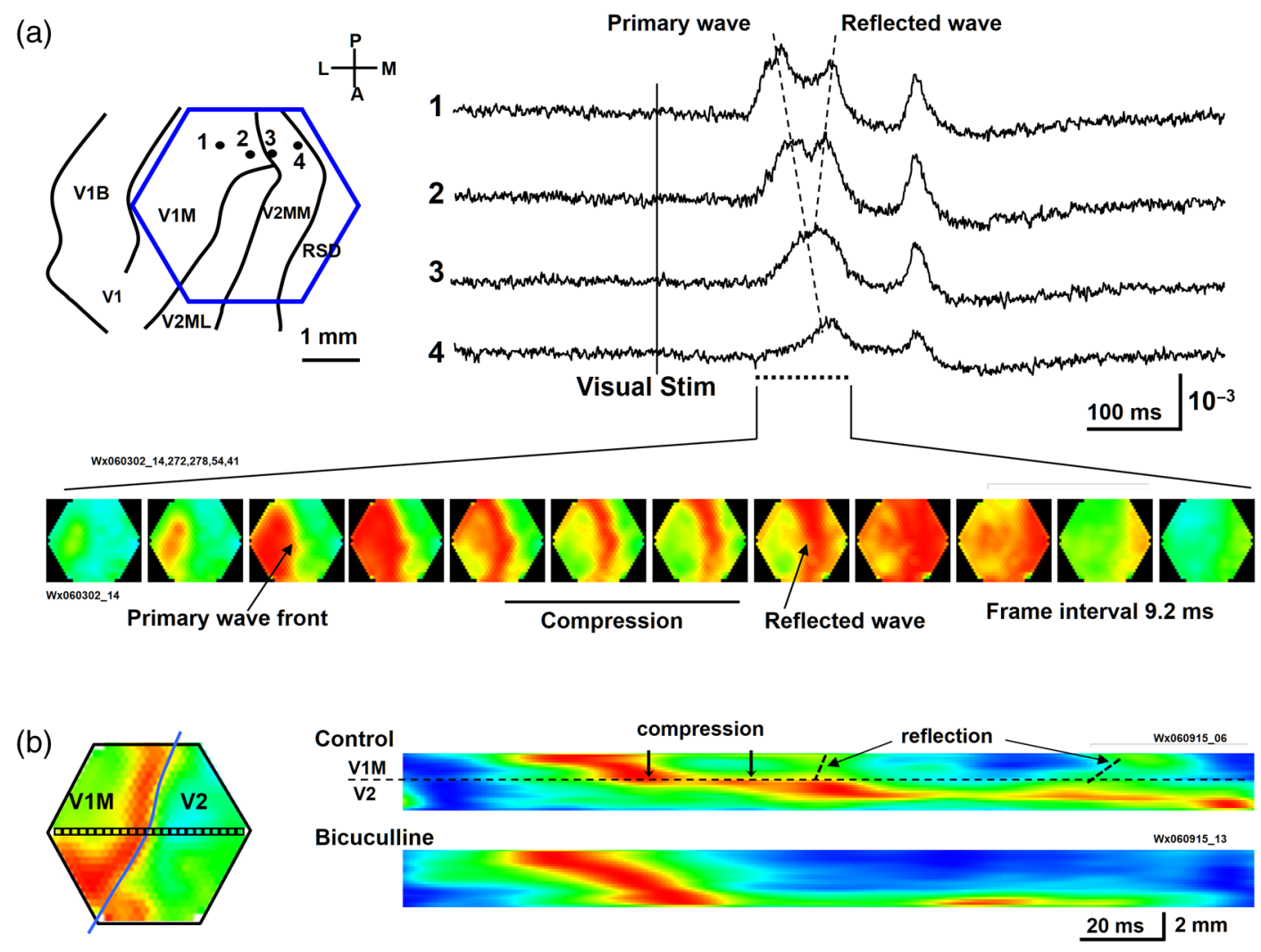

Fig. 5 Wave compression and reflection. (a) Left: Schematic drawing of imaging field (blue hexagon) overlying the map of the visual areas (left hemisphere, the map is shown as a mirror image of the cortex due to conversion in the macroscope). Four optical detectors, 1 to 4 , were selected (total of 464 detectors) and their signal traces are shown on the right. V1B, V1M: binocular and monocular areas of V1; V2MM, V2ML: mediomedial and mediolateral areas of V2; RSD: retrosplenial dysgranular. The map was made according to the stereotaxic map of Paxinos and Watson (2005). Right: Optical signals of visually evoked activity from four detectors (1 to 4 ). A drifting grating (onset time marked by the vertical line). The peak of the activity occurred sequentially from detector 1 to 4 , indicating a forward propagating wave (primary wave) from V1 to V2 (left broken line). A reflected wave can be seen starting from detector 3 and propagating backward to detector 1 (right broken line). Bottom images: pseudocolor images (0.6-ms snap shots) of the initial section of the evoked response. Twelve images (time marked by the dotted line under the traces) are shown (from a total of 8192 frames in a 5-s recording trial). The first image was taken when the evoked primary wave first appeared in the V1 monocular area, $\sim 104 \mathrm{~ms}$ after the grating started to drift. (b) X-T map made from signals on the row of detectors (boxed in the left) showing the space-time of the activity across the V1/V2 border (dashed line). The thin stripe at the V1M/V2 border indicates that compression sustained for a long period. Note that $5-\mu \mathrm{M}$ bicuculline completely eliminated the wave compression. Modified from Ref. 7.

The wave and its reflection can be identified as double peaks even in individual detectors and in a single trial. [Fig. 5(a)]. While in different animals, the locations of the compression and the shape of the compression band varied, in the same animal, the compression band reliably occurred in the same location and the same shape from trial-to-trial. The similar compression/reflection pattern was observed under a variety of visual stimuli. The probability of evoking the wave decreased when either stimulus size or contrast was reduced; however, once the primary wave was initiated by suprathreshold stimulation, the same pattern of compression/reflection occurred.

The reliability of the wave compression suggests that it may be related to the neuroanatomical structure of the cortex, especially the border between V1 and V2. In rats, these bundles are abundant near the V1/V2 border. ${ }^{29}$ Electric shocks to the contralateral cortex activated the callosum fibers, and a shortlatency activation was seen in the VSD signals in the imaging cortex. The area of short-latency activation overlapped with the location of the wave compression band, further suggesting that the wave compression occurs the site of callosal fiber bundles. $^{7}$

Multiple wave compressions occurred along the path of propagation. A second compression often occurred medial to that of the first compression [Fig. 5(b)], probably at the border between mediomedial V2 (V2MM) and retrosplenial dysgranular areas. While the onset time of the second compression was more variable from trial-to-trial, the location of the second compression was fixed. Multiple compressions suggest that wave compression is associated with the border between cortical areas. ${ }^{7}$

The compression of the visually evoked wave appeared as an abrupt slowing of the wave leading edge. Before compression, the evoked waves propagated at 50 to $70 \mathrm{~mm} / \mathrm{s}$. When the wavefront reached the compression band, the velocity reduced to about $5 \mathrm{~mm} / \mathrm{s}$. Meanwhile, the trailing edge of the wave maintained a higher speed, pushing the slow wavefront into a thin band of compressed activity along the V1/V2 border [Fig. 5(b)]. The compression and the resulting thin band sustained for a relatively long time compared to the time taken 
(a)

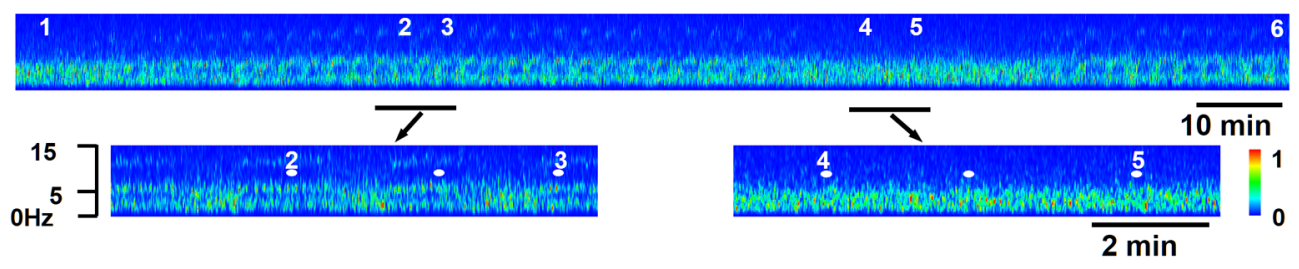

(b)

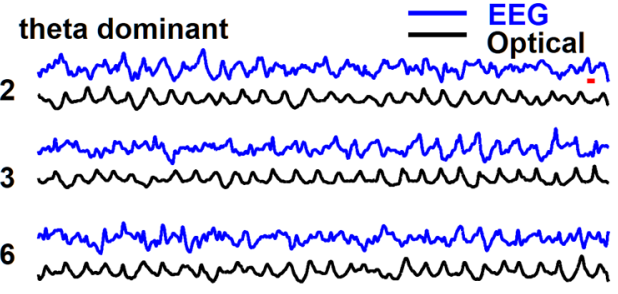

(c) delta dominant

1

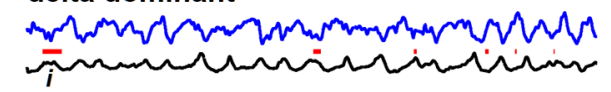

4

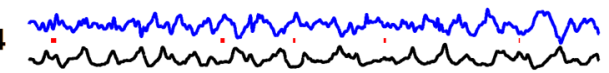
ii

5

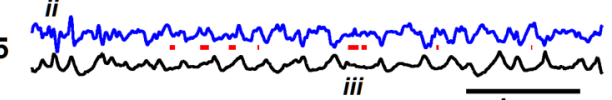

I $0.5 \mathrm{mV} \quad$ I $0.1 \% \mathrm{DF} / \mathrm{F}$ (d)

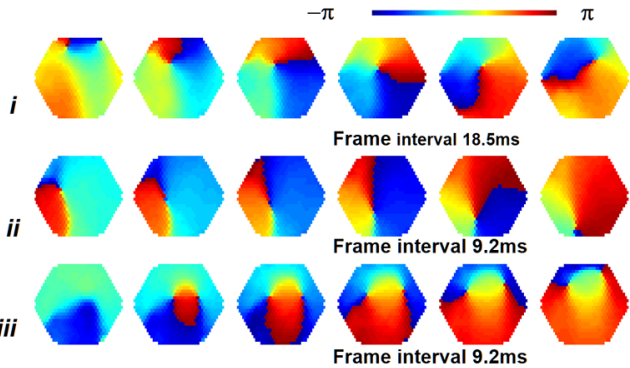

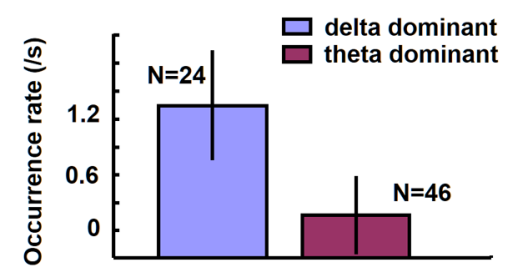

(e)

Fig. 6 Spiral waves during sleep. (a) EEG spectrum, showing alternation of theta and delta states. (b) and (c) Simultaneous EEG (blue) and optical (black) traces recorded during the theta-dominant [(b), trials 2, 3, and 6] and delta-dominant periods [(c), trials 1, 4, and 5]. Spiral waves were identified from the 8192 images of each trial and durations of spiral waves were marked by red lines under each EEG trace. Three example spiral waves (indicated by i, ii, and iii) are shown in panel (d). (d) Phase maps of example spiral waves in sleep-like states. Note that the bottom row images show an example of double spirals with opposite rotating directions. The imaging area is $4 \mathrm{~mm}$ in diameter. (e) Rate of occurrence of spiral waves during sleep-like states. Modified from Ref. 8.

for the activity expanding within V1. Inhibition in local circuits may play a role in the control of velocity. Bicuculline, even with a low concentration ( 3 to $5 \mu \mathrm{M}$ ), completely abolished the wave compression without significantly changing the speed of wave propagation within V1 [Fig. 5(b), bottom right graph].

In the visual cortex, both evoked and spontaneous cortical activities manifested as propagating waves. The spatiotemporal pattern of evoked and spontaneous events differed markedly. The evoked waves were initiated in V1, compressed near the V1/V2 border, and had a reflected wave. By contrast, spontaneous waves initiated at different locations did not show compression and reflection at the V1/V2 border. Imaging from the same field of view in the visual cortex found that compression/ reflection patterns occurred only in evoked but not in spontaneous waves, ${ }^{7}$ suggesting that the compression and reflection are governed by mechanisms specific for flow of information related to visual inputs.

\section{Spiral Waves in Mammalian Cortex}

Spiral waves are self-sustained, regenerative activity. ${ }^{30}$ Spirals rotate around a center point known as a rotor. A spiral rotor can emerge from free-ends of a traveling wavefront. The rotor, after being created, becomes a powerful organizer of energy (as seen in hurricane systems) by sending robust rotating waves outward (as seen in cardio arrhythmia). ${ }^{31,32}$ Similarly, in the brain, spiral waves may provide an essential mechanism for organizing the irregular activity of cortical neurons into rhythmic activity. ${ }^{8,33}$ Dominant local excitatory interactions are essential for sustaining spiral waves in general. ${ }^{30,31}$ In systems with extensive local excitatory connections, spiral waves are often seen, such as in the cardiac muscle during arrhythmia. ${ }^{34}$ In rodent brain slices, where local excitatory connections are dominant, spirals can be sustained for a long time. ${ }^{35}$ Similarly, spirals are also observed in the awake turtle visual cortex during the visual process. ${ }^{36}$ In the cortex in vivo, long-range and nonlocal connections, such as thalamocortical and corticocortical connections, may disrupt the spiral rotors and make spiral waves unsustainable. ${ }^{35}$ Therefore, spiral waves in intact cortex may occur during certain brain states when local excitatory interactions are predominant, while they are unsustainable in other brain states when long-range connections are strong.

The pulsation artifact is the major technical difficulty for examining spiral waves in mammalian cortex. The pulsation rate is similar to the spiral rotation rate, making it difficult to be isolated from the signal. In addition, spatial and temporal averaging cannot be used, due to the small size of the spiral center (the singularity) and irregular rate of the rotating wave. ${ }^{35}$ Blue dyes are so far the only dyes available for examining spiral dynamics in vivo.

Spirals were frequently seen (about $40 \%$ of all recording traces) during sleep-like states and cortical oscillations induced by carbachol and bicuculline. ${ }^{8,35}$ Spirals occurred alternately with other wave patterns (Fig. 6). In the cortex, most spiral waves $(81 \%)$ were short-lived (1-3 rotations), in contrast to 
(a) Sleep-like state $-\pi+\pi$

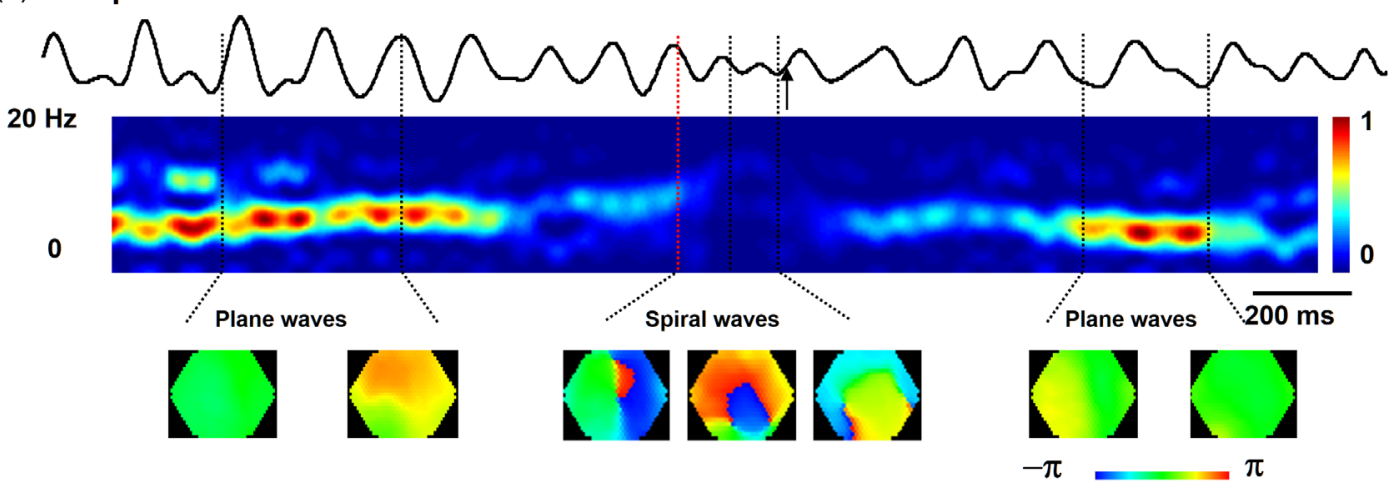

(b) in vivo (sleep-like)

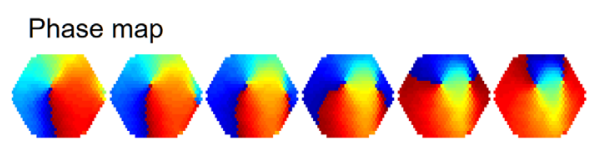

Hilbert amplitude

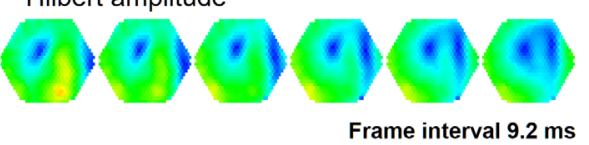

(c) in brain slices
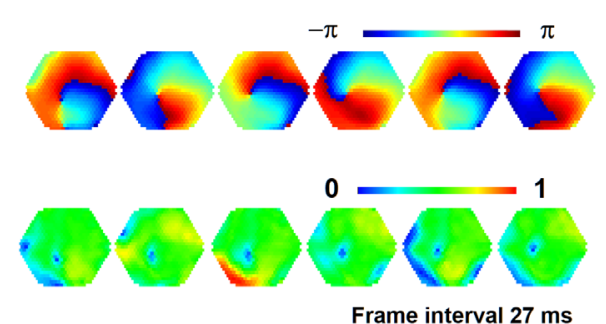

Fig. 7 Frequency attraction and power reduction during spirals. (a) Top trace: Raw VSD signal from an optical detector near the spiral center. Middle image: frequency-time map (frequency spectrum) made from that detector. Bottom images: selected from the phase movie for identifying wave patterns. Broken vertical lines indicate the times for these images. The red broken lines mark the first cycle of spiral waves and black arrows mark the end of spiral waves. (b) and (c) Examples from spiral waves during sleep-like states and in brain slices. The top row images are phase maps, identifying the location of the singularity. The bottom row images are the Hilbert amplitude, each from the same moment as the phase map in the top row. The blue area surrounding the phase singularity indicates the range of amplitude reduction. Note that the area of amplitude reduction is larger for in vivo spirals. Modified from Ref. 8.

the long lasting spirals seen in cardiac tissue and in brain slices. ${ }^{35}$ Spiral waves have spatiotemporal patterns distinct from other waves, such as target and plane waves. The best way to present spirals is the phase map of the VSD signal [Fig. 6(d)].

Rodent sleep contains an alternation of theta/delta states in continuous EEG recordings. The alternation is clearly seen in the frequency spectrum with time [the F/T map, Fig. 6(a)]. Using EEG frequency as an indicator, intermittent optical imaging trials were performed during the theta or delta dominant states [Figs. 6(b) and 6(c)]. Image frames were composed from the 464 channels of VSD signals, and phase maps were generated from the Hilbert transformation of the time series of the imaging frames (described in detail in Refs. 8 and 35). The occurrence rate of spiral waves was higher during the deltadominant state than during the theta-dominant state [Fig. 6(e)].

In about $40 \%$ of cases, spirals occurred in pairs [Fig. 6(d) iii, Fig. 7(b)], and the two spirals rotated in opposite directions. Breaking of the plane wavefront is a possible mechanism for generating these double spirals. At the broken end of the plane wave, excitation propagates forward and sideways, resulting in a slow combined velocity. The velocity continues slowing down with time, causing a curvature of wavefront near the broken end and developing into a rotor [see Fig. 8(c) in Ref. 8].

The emergence of spiral waves corresponded with changes in the frequency and power of cortical activity (Fig. 7). The changes were very obvious, even when the spirals were shortlived. To quantify the spatial profile of the amplitude reduction, we employed the Hilbert transform to estimate the "instantaneous amplitude" (Hilbert amplitude), so that the modulation of the phase was excluded. ${ }^{8,35}$ Hilbert amplitude maps in Figs. 7(b) and 7(c) clearly showed areas with amplitude reduction (blue) surrounding the phase singularities, demonstrating that the reduction in the amplitude was real, and not the result of phase modulation. The profiles of amplitude reduction showed significant differences between the in vitro and in vivo conditions: in slices, the amplitude reduction was limited to the spiral center [Fig. 6(c) in Ref. 35], while in in vivo, a larger area of amplitude reduction was seen [Fig. 7(b), bottom row]. Amplitude reduction surrounding a phase singularity is the hallmark of true spiral waves. ${ }^{30}$ During pentobarbital induced sleep-like states, an increased frequency, from sleep-like theta $(\sim 4 \mathrm{~Hz})$ to the rotation rate of the spiral $[\sim 10 \mathrm{~Hz}$, Fig. 7(a)] further suggests that the rotating waves were true spirals. Elevation in frequency during spirals appeared not to be caused by a high level of neuronal activity that overwhelmed the system. Instead, total power was reduced near the center [Fig. 7(a), center], suggesting that the spiral singularity was a dynamically stable event, which recruits neurons into its dynamical regime without increasing the power.

We propose that cortical spiral waves are not generated by specialized "spiral circuits," but instead, by a dynamic mechanism involved in the propagation of an excitation wave. In this sense, the process for generating cortical spirals is similar to that involved in spiral generation in the excitatory continua during 
Belousov-Zhabotinsky reactions, ${ }^{37}$ calcium waves on the surface of fertilizing Xenopus oocytes, ${ }^{38}$ and spreading depression waves in the retina. ${ }^{39}$ It is known in several biological systems that once a spiral rotor (phase singularity) is generated, it is protected from external disruptions by this strong interaction of surrounding rotating waves. ${ }^{31,32,35}$ This unique protection of the rotor makes it difficult to extinguish sustained spiral waves, which leads to harmful re-entry patterns underlying cardiac fibrillation. ${ }^{34}$ However, the situation in the cortex is different. Cortical networks contain both local and long-range connections, resembling a network structure with "small world" topography. ${ }^{40,41}$ We hypothesize that modulations arising from a third dimension by long-range thalamocortical and corticocortical connections may change the excitability of the local network, and by doing so, control the emergence, drifting, and extinction of spiral waves.

In an intact brain, subcortical generators can be strong and dominate cortical rhythms. ${ }^{42-44}$ In rodent, thalamocortical delta and hippocampal theta rhythms are dominant during different phases of sleep. ${ }^{45} \mathrm{We}$ found that the occurrence of spiral waves is strongly correlated to the cortical rhythms (Fig. 6).

As shown in Fig. 7, the formation of spirals reduced the power, changed the frequency to spiral rotation rate, and changed the spatiotemporal patterns from plane to rotation wave. These changes may allow spiral dynamics to have an influence in cortical processing by organizing neuronal activity over space.

While our understanding of the functional relevance of cortical spatiotemporal patterns is in its early stage, spiral waves provide a dynamic pattern that emerges from cortical population interactions and may have a powerful influence on the cortical oscillations and cortical processing.

\section{Conclusion}

Among many distinct contributions made by Amiran Grinvald and colleagues, the invention of blue dyes provided an excellent tool for imaging cortical dynamics, about 15 years before the first useful genetically encoded voltage florescent sensors became practically useable. Blue dyes offer excellent signalto-noise ratio, low pulsation artifacts, and low phototoxicity, allowing the use of a high dynamic range imaging devices to achieve high-sensitivity recordings comparable to that of LFP recordings. Blue dyes will continuously be a useful tool to examine neuronal population dynamics in the cortex when multitrial averaging is not possible.

\section{Disclosures}

The authors have no relevant financial interests in the manuscript and no other potential conflicts of interest to disclose. All surgical procedures were approved by Georgetown University Animal Care and Use Committee, strictly following $\mathrm{NIH}$ recommendations and guidelines.

\section{Acknowledgments}

This work was supported by an NIH grant (NS059034) to J. Y. W., and a Chinese NSFC grant (61302035) to X. G.

\section{References}

1. D. Shoham et al., "Imaging cortical dynamics at high spatial and temporal resolution with novel blue voltage-sensitive dyes," Neuron 24(4), 791-802 (1999).
2. M. T. Lippert et al., "Methods for voltage sensitive dye imaging of rat cortical activity with high signal to noise ratio," J. Neurophysiol. 98(1), 502-512 (2007).

3. H. Slovin et al., "Long-term voltage-sensitive dye imaging reveals cortical dynamics in behaving monkeys," J. Neurophysiol. 88(6), 3421-3438 (2002).

4. C. C. H. Petersen, A. Grinvald, and B. Sakmann, "Spatiotemporal dynamics of sensory responses in layer $2 / 3$ of rat barrel cortex measured in vivo by voltage-sensitive dye imaging combined with whole-cell voltage recordings and neuron reconstructions," J. Neurosci. 23(4), 1298-1309 (2003).

5. C. C. H. Petersen et al., "Interaction of sensory responses with spontaneous depolarization in layer 2/3 barrel cortex," Proc. Natl. Acad. Sci. U. S. A. 100(23), 13638-13643 (2003).

6. I. Ferezou, S. Bolea, and C. C. H. Petersen, "Visualizing the cortical representation of whisker touch: voltage-sensitive dye imaging in freely moving mice," Neuron 50(4), 617-629 (2006).

7. W. Xu et al., "Compression and reflection of visually evoked cortical waves," Neuron 55(1), 119-129 (2007).

8. X. Huang et al., "Spiral wave dynamics in neocortex," Neuron 68(5), 978-990 (2010).

9. X. Gao et al., "Interactions between two propagating waves in rat visual cortex," Neurosci. 216, 57-69 (2012).

10. D. Vitantonio et al., "Emergence of dominant initiation sites for interictal spikes in rat neocortex," J. Neurophysiol. 114(6), 3315-3325 (2015).

11. J. Liang et al., "Monitoring population membrane potential signals from neocortex," in Membrane Potential Imaging in the Nervous System and Heart, M. Canepari, D. Zecevic, and O. Bernus, Eds., Advances in Experimental Medicine and Biology, Vol. 859, pp. 171-196, Springer International Publishing, Switzerland (2015).

12. A. Benucci, R. A. Frazor, and M. Carandini, "Standing waves and traveling waves distinguish two circuits in visual cortex," Neuron 55(1), 103-117 (2007).

13. K. M. Dorries and J. S. Kauer, "Relationships between odor-elicited oscillations in the salamander olfactory epithelium and olfactory bulb," J. Neurophysiol. 83(2), 754-765 (2000).

14. I. Ferezou et al., "Spatiotemporal dynamics of cortical sensorimotor integration in behaving mice," Neuron 56(5), 907-923 (2007).

15. W. J. Freeman and J. M. Barrie, "Analysis of spatial patterns of phase in neocortical gamma EEGs in rabbit," J. Neurophysiol. 84(3), 1266-1278 (2000).

16. F. Han, N. Caporale, and Y. Dan, "Reverberation of recent visual experience in spontaneous cortical waves," Neuron 60(2), 321-327 (2008).

17. D. Jancke et al., "Imaging cortical correlates of illusion in early visual cortex," Nature 428(6981), 423-426 (2004).

18. Y. W. Lam et al., "Odors elicit three different oscillations in the turtle olfactory bulb," J. Neurosci. 20(2), 749-762 (2000).

19. J. A. London, L. B. Cohen, and J. Y. Wu, "Optical recordings of the cortical response to whisker stimulation before and after the addition of an epileptogenic agent," J. Neurosci. 9(6), 2182-2190 (1989).

20. E. V. Lubenov and A. G. Siapas, "Hippocampal theta oscillations are travelling waves," Nature 459 (7246), 534-539 (2009).

21. P. E. Roland et al., "Cortical feedback depolarization waves: a mechanism of top-down influence on early visual areas," Proc. Natl. Acad. Sci. U. S. A. 103(33), 12586-12591 (2006).

22. D. Rubino, K. A. Robbins, and N. G. Hatsopoulos, "Propagating waves mediate information transfer in the motor cortex," Nat. Neurosci. 9(12), 1549-1557 (2006).

23. D. M. Senseman and K. A. Robbins, "Modal behavior of cortical neural networks during visual processing," J. Neurosci. 19(10), RC3 (1999).

24. D. Sharon and A. Grinvald, "Dynamics and constancy in cortical spatiotemporal patterns of orientation processing," Science 295(5554), 512-515 (2002).

25. D. Sharon et al., "Cortical response field dynamics in cat visual cortex," Cereb. Cortex 17(12), 2866-2877 (2007).

26. H. Slovin et al., "Long-term voltage-sensitive dye imaging reveals cortical dynamics in behaving monkeys," J. Neurophysiol. 88(6), 3421-3438 (2002).

27. G. B. Ermentrout and D. Kleinfeld, "Traveling electrical waves in cortex: insights from phase dynamics and speculation on a computational role," Neuron 29(1), 33-44 (2001). 
28. J. Y. Wu, X. Huang, and C. Zhan, "Propagating waves of activity in the neocortex: what they are, what they do," Neuroscientist 14(5), 487-502 (2008).

29. J. F. Olavarria and R. Hiroi, "Retinal influences specify cortico-cortical maps by postnatal day six in rats and mice," J. Comp. Neurol. 459(2), 156-172 (2003).

30. A. T. Winfree, The Geometry of Biological Time, Springer-Verlag, New York (2001).

31. G. Bub, A. Shrier, and L. Glass, "Global organization of dynamics in oscillatory heterogeneous excitable media," Phys. Rev. Lett. 94(2), 028105 (2005).

32. J. M. Davidenko, "Stationary and drifting spiral waves of excitation in isolated cardiac muscle," Nature 355(6358), 349-351 (1992).

33. S. J. Schiff, X. Huang, and J. Y. Wu, "Dynamical evolution of spatiotemporal patterns in mammalian middle cortex," Phys. Rev. Lett. 98(17), 178102 (2007).

34. J. Jalife, "Rotors and spiral waves in atrial fibrillation," J. Cardiovasc. Electrophysiol. 14(7), 776-780 (2003).

35. X. Huang et al., "Spiral waves in disinhibited mammalian neocortex," J. Neurosci. 24(44), 9897-9902 (2004).

36. J. C. Prechtl et al., "Visual stimuli induce waves of electrical activity in turtle cortex," Proc. Natl. Acad. Sci. U. S. A. 94(14), 7621-7626 (1997).

37. J. P. Keener and J. J. Tyson, "Spiral waves in the Belousov-Zhabotinskii reaction," Phys. D 21, 307-324 (1986).

38. J. Lechleiter et al., "Spiral calcium wave propagation and annihilation in Xenopus laevis oocytes," Science 252(5002), 123-126 (1991).

39. N. A. Gorelova and J. Bures, "Spiral waves of spreading depression in the isolated chicken retina," J. Neurobiol. 14(5), 353-363 (1983).

40. S. H. Strogatz, "Exploring complex networks," Nature 410(6825), 268-276 (2001)
41. D. J. Watts and S. H. Strogatz, "Collective dynamics of 'small-world' networks," Nature, 393(6684), 440-442 (1998).

42. D. Contreras et al., "Control of spatiotemporal coherence of a thalamic oscillation by corticothalamic feedback," Science 274(5288), 771-774 (1996).

43. M. Steriade and I. Timofeev, "Neuronal plasticity in thalamocortical networks during sleep and waking oscillations," Neuron 37(4), 563-576 (2003).

44. M. Steriade, D. A. McCormick, and T. J. Sejnowski, "Thalamocortical oscillations in the sleeping and aroused brain," Science 262(5134), 679-685 (1993).

45. S. M. Montgomery, A. Sirota, and G. Buzsáki, "Theta and gamma coordination of hippocampal networks during waking and rapid eye movement sleep," J. Neurosci. 28(26), 6731-6741 (2008).

Xinling Geng received her $\mathrm{PhD}$ in biomedical engineering from the Department of Biomedical Engineering, Tsinghua University, in 2009. She is a lecturer of Capital Medical University, Beijing, China. She worked with Jian-young Wu at Georgetown University from 2013-2016. Her main research interest is spatiotemporal patterns of EEG and fast cortical waves revealed by voltage-sensitive dye imaging.

Jian-Young Wu received his $\mathrm{PhD}$ in biophysics from the Department of Biology, Peking University, in 1986. He is a professor of neuroscience at Georgetown University School of Medicine. He was mentored by Lawrence B. Cohen at Yale University from 1986-1993, and then joined Georgetown faculty in 1995 . His main research interest is neuronal population activity in the cortex. He uses voltage-sensitive dye imaging to study spatiotemporal patterns of the neuronal population events. 